\title{
A new unit root test against ESTAR based on a class of modified statistics
}

\section{Robinson Kruse}

\begin{abstract}
This paper proposes a new unit root test against a non-linear exponential smooth transition autoregressive (ESTAR) model. The new test is build upon the non-standard testing approach of Abadir and Distaso (2007) who introduce a class of modified statistics for testing joint hypotheses when one of the alternatives is one-sided. In a Monte Carlo study the popular Dickey-Fuller type test proposed by Kapetanios et al. (2003) is compared with the new test. The results suggest that the new test is generally superior in terms of power. An application to a real effective exchange rate underlines its usefulness.
\end{abstract}

Keywords Unit root test · Nonlinearities · Smooth transition

\section{Introduction}

Nonlinear time series models like the smooth transition autoregressive (STAR) model, see Teräsvirta (1994), have become very popular in the last years. In this paper we are mainly concerned with the exponential STAR (ESTAR) model and develop a new test for the unit root hypothesis against a globally stationary ESTAR model. In particular, we focus on a prominent and widely applied specification of this model that allows for a unit root regime and two symmetric mean-reverting regimes. The time series process, say $y_{t}$, behaves like a random walk if $y_{t-1}$ was close to some location parameter $c$ and it is meanreverting if $y_{t-1}$ departs from $c$. In the exponential smooth transition model the degree of mean-reversion depends on the squared difference between $y_{t-1}$ and $c$. When modeling real exchange rates for example, the economic intuition behind this specification is that the real exchange rate is nonstationary if it was

Robinson Kruse

Königsworther Platz 1, D-30167 Hannover

Tel.: +49-511-7625636

Fax: +49-511-7623923

E-mail: kruse@statistik.uni-hannover.de 
quite close its long run equilibrium value in the last period and that there are driving forces like arbitrage that leads to mean-reversion if the real exchange rate departs from its long run equilibrium. Moreover, arbitrage may not be profitable if the departure is small. Therefore, the degree of mean-reversion is small as well and vice versa. These facts make this ESTAR specification quite attractive for modeling economic time series like real exchange and interest rates, unemployment rates and $\log$ dividend yields.

There are a lot of economic theories like Purchasing Power Parity (PPP), to name a highly debated one, that imply certain time series properties, i.e. the stationarity of real exchange rates. Often the unit root hypothesis, which contradicts PPP, is tested against stationarity with extant linear unit root tests. These tests have however not the highest power when the true data generating process exhibits nonlinearities. Therefore, many recent empirical studies make use of nonlinear unit root tests.

Regarding the ESTAR specification from above, a popular Dickey-Fuller type test has been proposed by Kapetanios et al. (2003). However, this test assumes that the location parameter $c$ in the smooth transition function is equal to zero. On the contrary, a lot of empirical studies on real exchange rates report significant estimates of $c$, cf. Michael et al. (1997), Sarantis (1999), Taylor et al. (2001) and more recently, Rapach and Wohar (2006). When relaxing this assumption, we are faced with a non-standard testing problem, i.e. a joint hypothesis where one parameter is one-sided under the alternative while all others are two-sided. Since standard inference techniques are not appropriate in this situation, we make use of the new approach by Abadir and Distaso (2007) who propose a class of modified test statistics in order to tackle such non-standard testing problems. Our aim is to derive a unit root test allowing for a nonzero location parameter $c$ that can compete with the extant one of Kapetanios et al. (2003) in terms of power.

After introducing the ESTAR specification in more detail and presenting the existing test by Kapetanios et al. (2003) in section 2, the inference techniques by Abadir and Distaso (2007) and the new test are discussed in section 3 . The non-standard limiting distribution of the test statistic is derived and consistency of the test is proven. Moreover, we show that the limiting distribution remains unchanged if we account for potential serial correlation in the error terms by augmenting the test regression with lags of the dependent variable. By means of a Monte Carlo study in section 4 we compare the small sample properties of both tests under a variety of conditions. The new test is correctly sized and quite often superior in terms of power. Both tests have lower but substantial power when the true data generating process is a logistic STAR model. However, the new test has generally higher power against logistic STAR models than the extant test. Finally, we provide an application to a monthly real effective exchange rate time series for the European Union in section 5. The results suggest the validity of PPP if the new test is used and the opposite if the extant test is applied. Proofs are given in the appendix. 


\section{ADF-type unit root test against ESTAR}

The ESTAR specification we are concerned with is formally given by

$$
\triangle y_{t}=\alpha y_{t-1}+\phi y_{t-1}\left(1-\exp \left\{-\gamma\left(y_{t-1}-c\right)^{2}\right\}\right)+\varepsilon_{t},
$$

where $\varepsilon_{t} \sim \operatorname{iid}\left(0, \sigma^{2}\right)$. If the smoothness parameter $\gamma$ approaches zero, the ESTAR model becomes a linear $\mathrm{AR}(1)$ model, i.e. $\triangle y_{t}=\alpha y_{t-1}+\varepsilon_{t}$ that is stationary if $-2<\alpha<0$. In the following, $\alpha$ is set equal to zero which means that the ESTAR model becomes a random walk if $\gamma=0$. Kapetanios et al. (2003) show that the ESTAR model under the restriction $\alpha=0$,

$$
\triangle y_{t}=\phi y_{t-1}\left(1-\exp \left\{-\gamma\left(y_{t-1}-c\right)^{2}\right\}\right)+\varepsilon_{t},
$$

is globally stationary if $-2<\phi<0$ is true although it is locally non-stationary in the sense that it contains a partial unit root when $y_{t-1}=c$ holds. Additionally note that the random walk model can also be achieved when imposing the restriction $\phi=0$. This means that a direct test for the unit root hypothesis is infeasible since $\phi$ is not identified when testing $H_{0}: \gamma=0$ and vice versa. A popular approach to avoid the presence of nuisance parameters under the null hypothesis is to use a Taylor approximation of the smooth transition function $\left.G\left(y_{t-1} ; \gamma, c\right)=1-\exp \left\{-\gamma\left(y_{t-1}-c\right)^{2}\right\}\right)$ around $\gamma=0$, see Luukkonen et al. (1988). This approach was adopted by Kapetanios et al. (2003) and we construct the new test on the same basis. More specifically, Kapetanios et al. (2003) make the restriction $c=0$ and consider the model

$$
\triangle y_{t}=\phi y_{t-1}\left(1-\exp \left\{-\gamma y_{t-1}^{2}\right\}\right)+\varepsilon_{t} .
$$

An application of a first-order Taylor approximation leads to the auxiliary regression

$$
\triangle y_{t}=\beta_{1} y_{t-1}^{3}+u_{t},
$$

with $u_{t}$ being a noise term depending on $\varepsilon_{t}, \phi$ and the remainder of the Taylor expansion. Obviously, it looks very much like the famous Dickey-Fuller test regression without deterministic terms. The cubic term $y_{t-1}^{3}$ approximates the ESTAR nonlinearity.

The authors suggest a $t$-test for the unit root hypothesis against globally stationary ESTAR which corresponds to $H_{0}: \beta_{1}=0$ versus $H_{1}: \beta_{1}<0$. Hence, the unit root test is carried out by estimating the auxiliary regression (1) and computing a Dickey-Fuller type $t$-test, labeled as KSS,

$$
\mathrm{KSS} \equiv \frac{\hat{\beta}_{1}}{\sqrt{\operatorname{vâr}\left(\hat{\beta}_{1}\right)}}=\frac{\sum_{t=1}^{T} y_{t-1}^{3} \triangle y_{t}}{\sqrt{\hat{\sigma}^{2} \sum_{t=1}^{T} y_{t-1}^{6}}},
$$

where $\hat{\sigma}^{2}=\frac{1}{T} \sum_{t=1}^{T}\left(\triangle y_{t}-\hat{\beta}_{1} y_{t-1}^{3}\right)^{2}$ is the usual estimator of the error variance. Let $W(r)$ be the Brownian motion defined on $r \in[0,1]$ and let $\Rightarrow$ denote 
convergence in distribution. The limiting distribution of the KSS statistic is then given by

$$
\mathrm{KSS} \Rightarrow \frac{\frac{1}{4} W(1)^{4}-\frac{3}{2} \int_{0}^{1} W(r)^{2} d r}{\left(\int_{0}^{1} W(r)^{6}\right)^{1 / 2}}
$$

see Theorem 1 in Kapetanios et al. (2003). Regarding deterministic terms, they suggest to demean or detrend the data in a first step, i.e.

$$
y_{t}=\omega^{\prime} d_{t}+v_{t}
$$

with $d_{t}=1$ or $d_{t}=\left[\begin{array}{ll}1 & t\end{array}\right]^{\prime}$ and $\omega$ is a parameter vector of suitable dimension. In a second step, the unit root test is applied to $\widehat{v}_{t}$. As a consequence, the asymptotic distribution of the KSS statistic depends on functionals of a demeaned or detrended Brownian motion, respectively. The demeaned and detrended Brownian motion are given by

$$
\begin{aligned}
& W(r)-\int_{0}^{1} W(r) d r \\
& W(r)+(6 r-4) \int_{0}^{1} W(r) d r+(12 r-6) \int_{0}^{1} r W(r) d r,
\end{aligned}
$$

respectively. For details concerning this test such as proofs and critical values see Kapetanios et al. (2003).

\section{Modified Wald Type test}

In order to allow for a nonzero location parameter $c$ in the exponential transition function we consider the nonlinear time series model

$$
\triangle y_{t}=\phi y_{t-1}\left(1-\exp \left\{-\gamma\left(y_{t-1}-c\right)^{2}\right\}\right)+\varepsilon_{t} .
$$

Following Kapetanios et al. (2003), we apply a first-order Taylor approximation to $G\left(y_{t-1} ; \gamma, c\right)=\left(1-\exp \left\{-\gamma\left(y_{t-1}-c\right)^{2}\right\}\right)$ around $\gamma=0$ and proceed with the test regression

$$
\triangle y_{t}=\beta_{1} y_{t-1}^{3}+\beta_{2} y_{t-1}^{2}+\beta_{3} y_{t-1}+u_{t} .
$$

Following Kapetanios et al. (2003) we impose $\beta_{3}=0$ to improve the power of the test, see Kapetanios et al. (2003), footnote 5. Henceforth, we proceed with

$$
\triangle y_{t}=\beta_{1} y_{t-1}^{3}+\beta_{2} y_{t-1}^{2}+u_{t} .
$$

where $\beta_{1}=\gamma \phi$ and $\beta_{2}=-2 c \gamma \phi$. We are interested in the pair of hypotheses given by $H_{0}: \gamma=0$ against $H_{1}: \gamma>0$. In the test regression (4), this pair of hypothesis is equivalent to $H_{0}: \beta_{1}=\beta_{2}=0$ against $H_{1}: \beta_{1}<0, \beta_{2} \neq 0$. Note that the two-sidedness of $\beta_{2}$ under $H_{1}$ stems from the fact that $c$ is allowed to take real values. This testing problem is non-standard in the sense that one parameter is one-sided under $H_{1}$ while the other one is two-sided. 
A standard Wald test would be inappropriate and we therefore apply the methods of Abadir and Distaso (2007) to derive a suitable test. In a nutshell, the one-sided parameter is orthogonalized with respect to the two-sided one. The modified Wald test builds upon the one-sided parameter $\left(\beta_{1}\right)$ and the transformed two-sided parameter, say $\beta_{2}^{\perp}$, that are stochastically independent by definition.

Let the parameter vector of the regression model (4) be $\theta=\left[\begin{array}{ll}\beta_{1} & \beta_{2}\end{array}\right]^{\prime}$. Following the notation of Abadir and Distaso (2007), the null hypothesis of a unit root is rewritten as

$$
H_{0}: h(\theta) \equiv\left[\begin{array}{ll}
h_{1}(\theta) & h_{2}(\theta)
\end{array}\right]^{\prime}=\left[\begin{array}{ll}
\beta_{1} & \beta_{2}
\end{array}\right]^{\prime}=\left[\begin{array}{ll}
0 & 0
\end{array}\right]^{\prime} .
$$

The alternative hypothesis of a globally stationary ESTAR model is given by

$$
H_{1}: h_{1}(\theta)<0 \text { or } h_{2}(\theta) \neq 0,
$$

which includes the subset hypothesis $H_{1}^{\bigcap}: h_{1}(\theta)<0$ and $h_{2}(\theta) \neq 0$. Theorem 6 in Abadir and Distaso (2007) states that the modified Wald test is consistent against $H_{1}$ as well as $H_{1}^{\bigcap}$. The standard Wald test statistic based on the Hessian matrix $\mathcal{H}$ is

$$
W_{\mathcal{H}}=h(\hat{\theta})^{\prime} V^{-1} h(\hat{\theta})
$$

where $V \equiv\left[\left.\left.\frac{\partial h(\theta)}{\partial \theta^{\prime}}\right|_{\theta=\hat{\theta}}(-\mathcal{H})^{-1} \frac{\partial h(\theta)^{\prime}}{\partial \theta}\right|_{\theta=\hat{\theta}}\right]$ with elements $v_{i j}$. In general, the modified Wald test statistic of Abadir and Distaso (2007) is given by

$$
\begin{aligned}
\tau= & \left(\left.\left.\frac{\partial h_{2 \cdot 1}(\theta)}{\partial \theta^{\prime}}\right|_{\theta=\hat{\theta}}(-\mathcal{H})^{-1} \frac{\partial h_{2 \cdot 1}(\theta)^{\prime}}{\partial \theta}\right|_{\theta=\hat{\theta}}\right)^{-1} \hat{h}_{2 \cdot 1}(\hat{\theta})^{2} \\
& +1\left(h_{1}(\hat{\theta})<0\right)\left(\left.\left.\frac{\partial h_{1}(\theta)}{\partial \theta^{\prime}}\right|_{\theta=\hat{\theta}}(-\mathcal{H})^{-1} \frac{\partial h_{1}(\theta)^{\prime}}{\partial \theta}\right|_{\theta=\hat{\theta}}\right)^{-1} \hat{h}_{1}(\hat{\theta})^{2},
\end{aligned}
$$

with $h_{2 \cdot 1}(\theta)$ being

$$
h_{2 \cdot 1}(\theta)=h_{2}(\theta)-\frac{h_{1}(\theta) v_{21}}{v_{11}} .
$$

The estimator of $h_{2 \cdot 1}(\theta)$ is simply given by $\hat{h}_{2 \cdot 1}(\hat{\theta})=h_{2}(\hat{\theta})-\frac{h_{1}(\hat{\theta}) \hat{v}_{21}}{\hat{v}_{11}}$. Based on these results, straightforward calculations lead us to

$$
\tau=\left(\hat{v}_{22}-\frac{\hat{v}_{21}^{2}}{\hat{v}_{11}}\right)^{-1}\left(\hat{\beta}_{2}-\hat{\beta}_{1} \frac{\hat{v}_{21}}{\hat{v}_{11}}\right)^{2}+1\left(\hat{\beta}_{1}<0\right) \frac{\hat{\beta}_{1}^{2}}{\hat{v}_{11}},
$$

which is the new test statistic for the unit root hypothesis against globally stationary ESTAR. A simpler and more intuitive way to formulate this statistic is

$$
\tau=t_{\beta_{2}^{\perp}=0}^{2}+1\left(\hat{\beta}_{1}<0\right) t_{\beta_{1}=0}^{2} .
$$

The two summands appearing in the test statistic $\tau$ can be interpreted as follows: the first term is a squared $t$-statistic for the hypothesis $\beta_{2}^{\perp} \equiv \beta_{2}-$ $\beta_{1} v_{21} / v_{11}=0$ with $\beta_{2}^{\perp}$ being orthogonal to $\beta_{1}$. Additionally, the second term is a squared $t$-statistic for the hypothesis $\beta_{1}=0$, the one-sidedness under $H_{1}$ 
Table 1 Critical values of the $\tau$ statistic

\begin{tabular}{rccc}
\hline & $d_{t}=0$ & $d_{t}=1$ & $d_{t}=\left[\begin{array}{ll}1 & t\end{array}\right]^{\prime}$ \\
\hline $1 \%$ & 13.15 & 13.75 & 17.10 \\
$5 \%$ & 9.53 & 10.17 & 12.82 \\
$10 \%$ & 7.85 & 8.60 & 11.10 \\
\hline \hline
\end{tabular}

is achieved by the multiplied indicator function. In the next step, the limiting distribution of $\tau$ is derived.

Assumption $1 y_{t}$ is a random walk, i.e. $y_{t}=y_{t-1}+\varepsilon_{t}$ with $y_{0}=0$ and $u_{t}$ being $\operatorname{iid}\left(0, \sigma^{2}\right)$ and $E\left|u_{t}\right|^{\delta}<\infty$ for $\delta \geq 6$.

In Theorem 1 we derive the asymptotic distribution of $\tau$ under the null hypothesis $H_{0}: \gamma=0$.

Theorem 1 Under assumption 1 the $\tau$ statistic has the following asymptotic distribution which is free of nuisance parameters:

$$
\tau \Rightarrow \mathcal{A}(W(r))+\mathcal{B}(W(r)),
$$

where $\mathcal{A}$ and $\mathcal{B}$ are functions of the Brownian motion $W(r)$ that are given in the proof. Under the alternative hypothesis $H_{1}: \gamma>0$ the $\tau$ statistic diverges with rate $T$.

Proof See Appendix.

We follow the approach of Kapetanios et al. (2003) and demean or detrend the data in a first step when allowing for deterministic terms. This means that the Brownian motion $W(r)$ appearing in the limiting distribution of the $\tau$ statistic has to be replaced by a demeaned or detrended Brownian motion, respectively.

Next, we consider the case of serially correlated errors. We allow for stationary linear innovations that are generated by a short-memory process $v_{t}$.

Assumption $1 \quad v_{t}=\psi(L) u_{t}=\sum_{j=0}^{\infty} \psi_{j} u_{t-j}$, where $\sum_{j=0}^{\infty} j\left|\psi_{j}\right|<\infty$ and $u_{t} \sim \operatorname{iid}\left(0, \sigma^{2}\right)$.

In Theorem 2 we show that the asymptotic distribution of the $\tau$ statistic does not change when adding a sum of lagged differences on the right hand side of the test regression, i.e.

$$
\triangle y_{t}=\beta_{1} y_{t-1}^{3}+\beta_{2} y_{t-1}^{2}+\sum_{i=1}^{p} \rho_{i} \triangle y_{t-i}+u_{t} .
$$

Theorem 2 The asymptotic distribution of the $\tau$ statistic does not change when the test regression in (5) is used instead of (4). 
Proof See Appendix.

Alternatively, one could consider more general error processes and derive a Phillips-Perron type test for the unit root hypothesis, see Rothe and Sibbertsen (2006) and Sandberg (2008). However, we focus on the augmented DickeyFuller version in this paper and leave the other for future research.

\section{Monte Carlo study}

This section covers the Monte Carlo study that compares the small sample performance of the new unit root test and the existing test by Kapetanios et al. (2003). Throughout this section we set the number of observations $T$ equal to 300 which is a reasonable sample size for many macroeconomic and financial time series like unemployment rates and interest rates. Furthermore, 500 initial observations are deleted to reduce the effect of initial conditions. Asymptotic critical values for the modified Wald-type test $\tau$ are provided in Table 1 . They are based on 20,000 replications and $T=1000$. We report critical values for raw $\left(d_{t}=0\right)$, demeaned $\left(d_{t}=1\right)$ and detrended data $\left(d_{t}=\left[\begin{array}{ll}1 & t\end{array}\right]^{\prime}\right)$ for nominal significance levels of one, five and ten percent, respectively.

We investigate the size of both tests under the following data generating processes

$$
\begin{aligned}
& y_{t}=y_{t-1}+\varepsilon_{t} \text { with } \varepsilon_{t}=u_{t} \\
& y_{t}=y_{t-1}+\varepsilon_{t} \text { with } \varepsilon_{t}=\rho \varepsilon_{t-1}+u_{t} \\
& y_{t}=y_{t-1}+\varepsilon_{t} \text { with } \varepsilon_{t}=u_{t}-\theta u_{t-1},
\end{aligned}
$$

where $u_{t}$ is drawn from the standard normal distribution. The errors $\varepsilon_{t}$ follow an iid process, an AR(1) or MA(1) process. We adopt the approach of Phillips and Sul (2003) and sample parameters from uniform distributions in order to cover a wide range of values in a relatively small number of experiments. Hence, the autoregressive and moving average parameters $\rho$ and $\theta$ are drawn from uniform distributions:

$$
\rho \sim U[\underline{\rho}, \bar{\rho}] \text { and } \theta \sim U[\underline{\theta}, \bar{\theta}] .
$$

We specify $\underline{\rho}=\underline{\theta}=0$ and $\bar{\rho}=\bar{\theta}=0.4$. The size experiments are based on 5,000 replications, results can be found in Table 2. Most rejection rates under the null hypothesis are quite close to the nominal ones which suggests that both tests are correctly sized. We observe that both tests are a little bit oversized in the presence of errors that follow a first-order moving average process.

Next, we study the power of both tests by considering different settings in the globally stationary nonlinear ESTAR process for the parameters $c$ and $\gamma$. The data generating process we consider under the nonlinear ESTAR alternative is

$$
\triangle y_{t}=\phi y_{t-1}\left(1-\exp \left\{-\gamma\left(y_{t-1}-c\right)^{2}\right\}\right)+\varepsilon_{t}
$$


Table 2 Size experiments

\begin{tabular}{|c|c|c|c|c|c|c|c|c|c|c|}
\hline \multirow[t]{2}{*}{$d_{t}$} & \multirow[t]{2}{*}{ Test } & \multicolumn{3}{|c|}{ iid } & \multicolumn{3}{|c|}{$\mathrm{AR}(1)$} & \multicolumn{3}{|c|}{ MA(1) } \\
\hline & & 1.0 & 5.0 & 10.0 & 1.0 & 5.0 & 10.0 & 1.0 & 5.0 & 10.0 \\
\hline \multirow[t]{2}{*}{0} & KSS & 0.9 & 5.1 & 9.9 & 1.0 & 5.0 & 10.0 & 1.2 & 4.7 & 10.1 \\
\hline & $\tau$ & 1.1 & 5.2 & 10.9 & 1.0 & 5.0 & 10.0 & 1.7 & 5.2 & 11.9 \\
\hline \multirow[t]{2}{*}{1} & KSS & 1.1 & 5.1 & 9.9 & 1.1 & 4.8 & 9.7 & 1.3 & 6.0 & 11.8 \\
\hline & $\tau$ & 1.1 & 4.9 & 9.8 & 0.9 & 4.6 & 9.5 & 1.2 & 5.6 & 11.8 \\
\hline \multirow[t]{2}{*}[\begin{array}{ll}{1}&{t}\end{array}]{$^{\prime}$} & KSS & 1.1 & 4.9 & 10.2 & 0.8 & 5.2 & 9.6 & 1.4 & 6.5 & 10.9 \\
\hline & $\tau$ & 1.0 & 5.2 & 10.1 & 0.9 & 5.4 & 10.3 & 1.5 & 6.6 & 11.2 \\
\hline
\end{tabular}

Notes: Reported values are rejection rates of KSS and $\tau$ test under the validity of $H_{0}$.

with $\phi=-1$. This restriction is often imposed in empirical studies, see for example Taylor et al. (2001). The location parameter $c$ is set either equal to zero or it is drawn from a uniform distribution with lower and upper bound, $\underline{c}$ and $\bar{c}$, respectively. Analogously, the smoothness parameter $\gamma$ is drawn from a uniform distribution with lower and upper bound $\underline{\gamma}$ and $\bar{\gamma}$, respectively:

$$
c \sim U[\underline{c}, \bar{c}] \text { and } \gamma \sim U[\underline{\gamma}, \bar{\gamma}] .
$$

Results of these power experiments are reported in the upper panel of Table 3 . In the first experiment we specify a zero location parameter $\left(c_{0} \equiv c=0\right)$ and slow transition between regimes $\left(\gamma_{l} \equiv \gamma \sim U[0.001,0.01]\right)$. In the second and third experiments a non-zero location parameter is allowed by drawing it from a uniform distribution with lower and upper bound of $-5(-10)$ and 5 (10), respectively. The fourth and fifth settings restrict the upper bound $\bar{c}$ to zero in order to have a non-zero mean of $c$. Please note that we do not report results for experiments where to the lower bound $\underline{c}$ is restricted to zero because there is no qualitative difference because of symmetry. The last two experiments are like the two previous ones but with fast transition between regimes, i.e. $\gamma_{h} \equiv \gamma \sim U[0.01,0.1]$.

When interpreting the simulated rejection probabilities against ESTAR we observe that the new test is generally superior to the Kapetanios et al. (2003) test in terms of power. Only in some cases where the unit root tests are applied to raw data $\left(d_{t}=0\right)$, the KSS test performs somewhat better than the modified Wald test. Most applications of unit root tests in economics involve deterministic terms. When data is demeaned or detrended, power gains up to 15 percent can be achieved by applying the new test.

In addition, we study the power of both tests against globally stationary logistic STAR (LSTAR) models. As noted by Kapetanios et al. (2003) an alternative nonlinear adjustment scheme to the exponential one is a logistic smooth transition function. We use the second-order logistic function

$$
G\left(y_{t-1} ; \gamma, c_{1}, c_{2}\right)=2 /\left[1+\exp \left(-\gamma\left(y_{t-1}-c_{1}\right)\left(y_{t-1}-c_{2}\right)\right)\right]-1
$$

that has two location parameters, namely $c_{1}$ and $c_{2}$. Like the exponential smooth transition function it becomes constant if $\gamma \rightarrow 0$, which means that 
Table 3 Power experiments

\begin{tabular}{|c|c|c|c|c|c|c|c|c|}
\hline$d_{t}$ & Test & $c_{0}, \gamma_{l}$ & $c_{ \pm 5}, \gamma_{l}$ & $c_{ \pm 10}, \gamma_{l}$ & $c_{-5}, \gamma_{l}$ & $c_{-10}, \gamma_{l}$ & $c_{-5}, \gamma_{h}$ & $c_{-10}, \gamma_{h}$ \\
\hline \multicolumn{9}{|c|}{ Exponential STAR } \\
\hline \multirow[t]{2}{*}{0} & KSS & 99.6 & 92.4 & 73.0 & 92.0 & 74.4 & 97.0 & 97.2 \\
\hline & $\tau$ & 95.3 & 88.1 & 72.6 & 88.5 & 74.5 & 98.2 & 97.9 \\
\hline \multirow[t]{2}{*}{1} & KSS & 91.1 & 87.6 & 77.8 & 88.4 & 79.7 & 98.1 & 97.5 \\
\hline & $\tau$ & 92.9 & 92.3 & 93.3 & 91.8 & 93.9 & 100 & 100 \\
\hline \multirow[t]{2}{*}[\begin{array}{ll}{1}&{t}\end{array}]{$^{\prime}$} & KSS & 77.5 & 74.2 & 64.1 & 73.4 & 65.9 & 97.7 & 96.3 \\
\hline & $\tau$ & 81.6 & 78.9 & 78.4 & 79.6 & 78.7 & 100 & 100 \\
\hline \multicolumn{9}{|c|}{ Logistic STAR } \\
\hline \multirow[t]{2}{*}{0} & KSS & 97.8 & 95.4 & 87.8 & 94.9 & 89.2 & 99.9 & 99.9 \\
\hline & $\tau$ & 83.2 & 82.5 & 82.7 & 80.3 & 83.3 & 99.9 & 100 \\
\hline \multirow[t]{2}{*}{1} & KSS & 75.6 & 72.4 & 63.7 & 71.4 & 64.6 & 99.0 & 94.4 \\
\hline & $\tau$ & 79.0 & 77.5 & 78.3 & 77.9 & 80.0 & 100 & 100 \\
\hline \multirow[t]{2}{*}[\begin{array}{ll}{1}&{t}\end{array}]{$^{\prime}$} & KSS & 53.3 & 50.4 & 46.5 & 50.8 & 48.5 & 97.5 & 92.3 \\
\hline & $\tau$ & 58.6 & 54.9 & 57.7 & 55.4 & 59.4 & 99.5 & 99.8 \\
\hline
\end{tabular}

Notes: Reported values are rejection rates of KSS test (upper entries) and $\tau$ test (lower entries). Nominal significance level is five percent.

the nonlinear logistic smooth transition model becomes linear. Without loss of generality, we set $c_{1}=0$ and draw $c_{2}$ from the uniform distribution as done before in the case of an exponential smooth transition. The data generating process is now given by

$$
\triangle y_{t}=y_{t-1}\left(1-2 /\left[1+\exp \left(-\gamma\left(y_{t-1}-c_{1}\right)\left(y_{t-1}-c_{2}\right)\right)\right]\right)+\varepsilon_{t} .
$$

Empirical rejection frequencies are reported in the lower panel of Table 3. Both tests have higher power against ESTAR than LSTAR models which is not surprising since both have the former one as specific alternative. Nonetheless, one might expect that both tests have substantial power against logistic STAR models because the Taylor approximation of a logistic STAR model is quite similar. Thus, a rejection of the null hypothesis does not necessarily contain information about the specific type of nonlinear adjustment. When comparing both unit root tests, we come to the same conclusions as before. In addition we observe that the power is lower for detrended data than for demeaned data which is due to an additional parameter that has to be estimated when detrending the data.

In sum, the new test shows good overall performance and is quite often more powerful than the existing test by Kapetanios et al. (2003), especially when the test is applied to demeaned or detrended data, which are the most important cases in practice.

\section{Empirical application}

Unit root tests have become a very popular tool in the literature that is concerned with testing validity of the Purchasing Power Parity (PPP) which counts to one of the most important parities in international macroeconomics. 


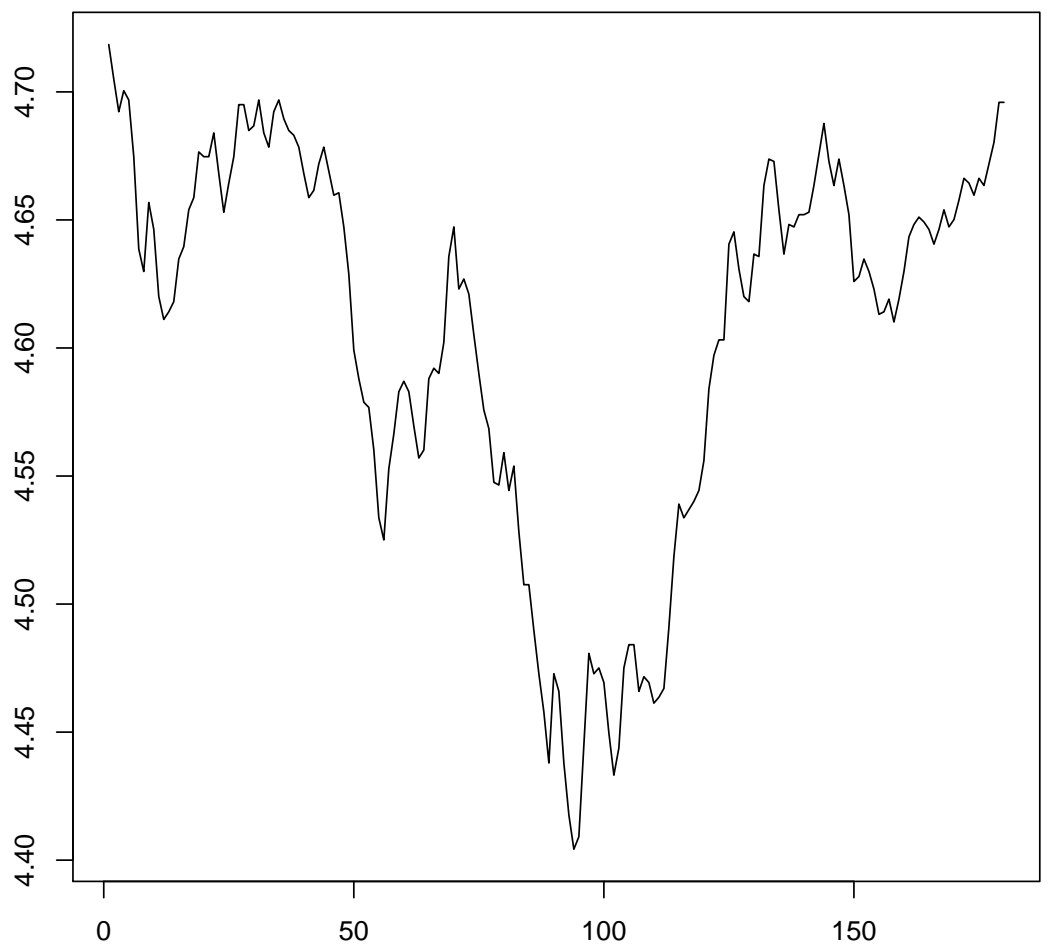

Fig. 1 Logarithm of real effective exchange rate (January, 1993 to December, 2007).

One can state that PPP holds if and only if the real exchange rate is stationary. Henceforth, testing the unit root hypothesis means testing the non-validity of the PPP theory. Since linear unit root tests like the ones of Dickey-Fuller (1979) and Phillips and Perron (1988) often fail to reject the null hypothesis of non-stationarity when being applied to real exchange rate data, researchers tend to use nonlinear unit root tests where the specific model that is true under the alternative is congruent with economic models of financial markets. For example, STAR models for the real exchange rate can be interpreted in the context of transaction costs and arbitrage, see Dumas (1992), Sercu et al. (1995) and Michael et al. (1997). However, rejecting the null hypothesis in favor of a nonlinear alternative while a linear Dickey-Fuller test does not reject in favor of a linear alternative might hint at nonlinearities. Nonetheless, one should be careful with the conclusion that nonlinearity is of ESTAR-type because the test regression approximates a lot of nonlinear models and such tests 
can have substantial power against other nonlinear mean-reverting processes, see section 4 .

We apply both unit root tests against nonlinear alternatives and two famous tests against linear alternatives to the monthly real effective exchange rate time series for the European Union. Our data is taken from Datastream (code: EMXTW..RF) and spans from 1993:01 to 2007:12 implying 180 observations. The logged time series is depicted in Figure 1. No linear trend can be seen in the data but the mean appears to be highly significant. Hence, we demean the data in a first step. In a second step we estimate the test regressions with a lag length chosen accordingly to the Schwarz information criterion $(\widehat{p}=1)$. We obtain $\mathrm{KSS}=-2.21$ which is not significant at the ten percent level suggesting that PPP does not hold. On the contrary, using the new test one has to reject the null hypothesis since $\tau=9.19$ is significant at the ten percent level which indicates that PPP holds true. Furthermore, the unit root tests against linear alternatives by Dickey and Fuller (1979) (DF) and Phillips and Perron (1988) (PP) do not provide any evidence against the null hypothesis. The test statistics are $\mathrm{DF}=-1.76$ and $\mathrm{PP}=-1.60$, respectively. We conclude that nonlinearities, potentially of exponential STAR-type with non-zero location, are present in the data but that they are not detected by applying existing tests. The modified Wald unit root test yields new evidence on the stationarity of the EU real effective exchange rate which suggests the validity of PPP.

\section{Conclusions}

This paper contributes to the literature on nonlinear unit root tests by generalizing the existing test by Kapetanios et al. (2003) with respect to a nonzero location parameter. The resulting non-standard testing problem is tackled by deriving a modified Wald test that builds up on the inference techniques by Abadir and Distaso (2007). The non-standard limiting distribution of the test statistic has been derived under standard assumptions. The Monte Carlo study shows that the new test is in most situations superior to the extant test. An empirical application to the EU real effective exchange rate underpins its usefulness.

\section{Appendix}

Proof (of Theorem 1) In order to simplify the notation, we write $\int$ instead of $\int_{0}^{1}$ in the following. The proof makes use of the following convergence results, see Hansen (1992) and Hamilton (1994). We have

$$
\frac{1}{T^{(i+2) / 2}} \sum_{t=1}^{T} y_{t-1}^{i} \Rightarrow \sigma^{i} \int W(r)^{i} d r \quad \text { for } i=4,5,6
$$




$$
\begin{aligned}
\frac{1}{T^{3 / 2}} \sum_{t=1}^{T} y_{t-1}^{2} \triangle y_{t} & \Rightarrow \sigma^{3}\left(\frac{1}{3} W(1)^{3}-\int W(r) d r\right) \\
\frac{1}{T^{2}} \sum_{t=1}^{T} y_{t-1}^{3} \triangle y_{t} & \Rightarrow \sigma^{4}\left(\frac{1}{4} W(1)^{4}-\frac{3}{2} \int W(r)^{2} d r\right) .
\end{aligned}
$$

We first note that the second summand of $\tau$ is given by $1\left(\hat{\beta}_{1}<0\right) t_{\beta_{1}=0}^{2}$ and that the OLS estimator for $\beta_{1}$ in (4) is given by

$$
\hat{\beta}_{1}=\frac{\sum_{t=1}^{T} y_{t-1}^{4} \sum_{t=1}^{T} y_{t-1}^{3} \triangle y_{t}-\sum_{t=1}^{T} y_{t-1}^{2} \triangle y_{t} \sum_{t=1}^{T} y_{t-1}^{5}}{\sum_{t=1}^{T} y_{t-1}^{4} \sum_{t=1}^{T} y_{t-1}^{6}-\left(\sum_{t=1}^{T} y_{t-1}^{5}\right)^{2}}
$$

Under the assumptions of Theorem 1 and by using the convergence results from above we obtain $\hat{\beta}_{1} \stackrel{P}{\rightarrow} \beta_{1}$ and $\hat{\beta}_{1}=O_{P}\left(T^{-2}\right)$. Furthermore,

$$
t_{\beta_{1}=0}=\frac{\sum_{t=1}^{T} y_{t-1}^{4} \sum_{t=1}^{T} y_{t-1}^{3} \Delta y_{t}-\sum_{t=1}^{T} y_{t-1}^{2} \Delta y_{t} \sum_{t=1}^{T} y_{t-1}^{5}}{\sqrt{\hat{\sigma}^{2}\left(\left(\sum_{t=1}^{T} y_{t-1}^{4}\right)^{2} \sum_{t=1}^{T} y_{t-1}^{6}-\sum_{t=1}^{T} y_{t-1}^{4}\left(\sum_{t=1}^{T} y_{t-1}^{5}\right)^{2}\right)}} .
$$

Again, by using the convergence results it follows that

$$
t_{\beta_{1}=0} \Rightarrow \frac{\left(\int W(r)^{4} d r\right)\left(\frac{1}{4} W(1)^{4}-\frac{3}{2} \int W(r)^{2} d r\right)-\left(\frac{1}{3} W(1)^{3}-\int W(r) d r\right)\left(\int W(r)^{5} d r\right)}{\sqrt{\left(\int W(r)^{4} d r\right)^{2}\left(\int W(r)^{6} d r\right)-\left(\int W(r)^{4} d r\right)\left(\int W(r)^{5} d r\right)^{2}}}
$$

and by applying the CMT it follows directly that $t_{\beta_{1}=0}^{2}$ converges to the square of the previous function which gives an expression for $\mathcal{B}(W(r))$. Regarding the first summand of $\tau$, we have for the nominator of $t_{\beta_{2}^{\perp}=0}$

$$
\hat{\beta}_{2}-\hat{\beta}_{1} \frac{\hat{v}_{21}}{\hat{v}_{11}}=\frac{\sum_{t=1}^{T} y_{t-1}^{4} \sum_{t=1}^{T} y_{t-1}^{6} \sum_{t=1}^{T} y_{t-1}^{2} \triangle y_{t}-\sum_{t=1}^{T} y_{t-1}^{2} \triangle y_{t}\left(\sum_{t=1}^{T} y_{t-1}^{5}\right)^{2}}{\left(\sum_{t=1}^{T} y_{t-1}^{4}\right)^{2} \sum_{t=1}^{T} y_{t-1}^{6}-\sum_{t=1}^{T} y_{t-1}^{4}\left(\sum_{t=1}^{T} y_{t-1}^{5}\right)^{2}} .
$$

For the denominator of $t_{\beta_{2}^{\perp}=0}$ we have after simple calculations

$$
\sqrt{\hat{v}_{22}-\frac{\hat{v}_{21}^{2}}{\hat{v}_{11}}}=\sqrt{\frac{\hat{\sigma}^{2}}{\sum_{t=1}^{T} y_{t-1}^{4}}} .
$$

Therefore

$$
t_{\beta_{2}^{\perp}=0}=\frac{\sum_{t=1}^{T} y_{t-1}^{2} \Delta y_{t}\left(\left(\sum_{t=1}^{T} y_{t-1}^{4}\right)^{3 / 2} \sum_{t=1}^{T} y_{t-1}^{6}-\left(\sum_{t=1}^{T} y_{t-1}^{4}\right)^{1 / 2}\left(\sum_{t=1}^{T} y_{t-1}^{5}\right)^{2}\right)}{\hat{\sigma}\left(\left(\sum_{t=1}^{T} y_{t-1}^{4}\right)^{2} \sum_{t=1}^{T} y_{t-1}^{6}-\sum_{t=1}^{T} y_{t-1}^{4}\left(\sum_{t=1}^{T} y_{t-1}^{5}\right)^{2}\right)}
$$


Using the convergence results stated above it follows that

$t_{\beta_{2}^{\perp}=0} \Rightarrow \frac{\left(\frac{1}{3} W(1)^{3}-\int W(r) d r\right)\left(\left(\int W(r)^{4} d r\right)^{3 / 2}\left(\int W(r)^{6} d r\right)-\left(\int W(r)^{4} d r\right)^{1 / 2}\left(\int W(r)^{5} d r\right)^{2}\right)}{\left(\int W(r)^{4} d r\right)^{2}\left(\int W(r)^{6} d r\right)-\left(\int W(r)^{4} d r\right)\left(\int W(r)^{5} d r\right)^{2}}$

Again, by CMT it follows that $t_{\beta_{2}^{\perp}=0}^{2}$ converges in distribution to the square of the previous function which gives an expression for $\mathcal{A}(W(r))$. It is easy to show that $\hat{\sigma}^{2} \stackrel{p}{\rightarrow} \sigma^{2}$, see Kapetanios et al. (2003). Under the alternative hypothesis, $\triangle y_{t}, y_{t-1}^{2}$ and $y_{t-1}^{3}$ are $I(0)$ processes and it is easy to show that the terms appearing in the test statistic are $O_{P}(T)$. Then, $t_{\beta_{2}^{\perp}=0}^{2}=O_{P}(T)$ and $t_{\beta_{1}=0}^{2}=O_{P}(T)$, therefore $\tau=O_{P}(T)$. The $\tau$ statistic is therefore diverging with rate $T$.

Proof (of Theorem 2) The proof is very similar to the one of Kapetanios et al. (2003) as it uses the same arguments. Let $Z=\left[\triangle y_{-1}, \triangle y_{-2}, \ldots, \triangle y_{-p}\right]$ with $\triangle y_{-i}=\left[\triangle y_{-i+1}, \triangle y_{-i+2}, \ldots, \triangle y_{T-i}\right]$ and $M_{T}=I_{T}-Z\left(Z^{\prime} Z\right)^{-1} Z$. Note that, $\hat{\sigma}^{2}=\frac{1}{T} \varepsilon^{\prime} M_{T} \varepsilon=\frac{1}{T} \varepsilon^{\prime} \varepsilon+o_{p}(1) \stackrel{p}{\rightarrow} \sigma^{2}$ with $\varepsilon=\left[\varepsilon_{1}, \varepsilon_{2}, \ldots, \varepsilon_{T}\right]^{\prime}$. Moreover, we have

$$
\begin{gathered}
\frac{1}{T^{(i+2) / 2}} y_{-1}^{i / 2^{\prime}} M_{T} y_{-1}^{i / 2}=\frac{1}{T^{(i+2) / 2}} y_{-1}^{i / 2^{\prime}} y_{-1}^{i / 2}+o_{p}(1) \Rightarrow \lambda^{i} \int W(r)^{i} d r \text { for } i=4,6 \\
\frac{1}{T^{7 / 2}} y_{-1}^{5 / 2^{\prime}} M_{T} y_{-1}^{5 / 2}=\frac{1}{T^{7 / 2}} y_{-1}^{5 / 2^{\prime}} y_{-1}^{5 / 2}+o_{p}(1) \Rightarrow \lambda^{5} \int W(r)^{5} d r,
\end{gathered}
$$

and additionally,

$$
\begin{aligned}
\frac{1}{T^{3 / 2}} y_{-1}^{2^{\prime}} M_{T} \varepsilon & =\frac{1}{T^{3 / 2}} y_{-1}^{2^{\prime}} \varepsilon+o_{p}(1) \\
\frac{1}{T^{2}} y_{-1}^{3^{\prime}} M_{T} \varepsilon & =\frac{1}{T^{2}} y_{-1}^{3^{\prime}} \varepsilon+\lambda^{3} W(1)^{3}-\lambda \sigma^{2} \int W(r) d r \\
& \Rightarrow \frac{1}{4} \lambda^{4} W(1)^{4}-\frac{3}{2} \sigma^{2} \lambda^{2} \int W(r)^{2} d r,
\end{aligned}
$$

where $\lambda^{2}$ is the long-run variance of $\triangle y_{t}$ under the null hypothesis. Based on these results we have

$$
\begin{aligned}
t_{\beta_{1}=0} & =\frac{\left(y_{-1}^{2^{\prime}} M_{T} y_{-1}^{2}\right)\left(y_{-1}^{3^{\prime}} M_{T} \varepsilon\right)-\left(y_{-1}^{2^{\prime}} M_{T} \varepsilon\right)\left(y_{-1}^{5 / 2^{\prime}} M_{T} y_{-1}^{5 / 2}\right)}{\sqrt{\hat{\sigma}^{2}\left(\left(y_{-1}^{2^{\prime}} M_{T} y_{-1}^{2}\right)^{2}\left(y_{-1}^{3^{\prime}} M_{T} y_{-1}^{3}\right)-\left(y_{-1}^{2^{\prime}} M_{T} y_{-1}^{2}\right)\left(y_{-1}^{5 / 2^{\prime}} M_{T} y_{-1}^{5 / 2}\right)^{2}\right)}} \\
& =\frac{\left(y_{-1}^{2^{\prime}} y_{-1}^{2}\right)\left(y_{-1}^{3^{\prime}} \varepsilon\right)-\left(y_{-1}^{2^{\prime}} \varepsilon\right)\left(y_{-1}^{5 / 2^{\prime}} y_{-1}^{5 / 2}\right)}{\sqrt{\hat{\sigma}^{2}\left(\left(y_{-1}^{2^{\prime}} y_{-1}^{2}\right)^{2}\left(y_{-1}^{3^{\prime}} y_{-1}^{3}\right)-\left(y_{-1}^{2^{\prime}} y_{-1}^{2}\right)\left(y_{-1}^{5 / 2^{\prime}} y_{-1}^{5 / 2}\right)^{2}\right)}}+o_{p}(1) .
\end{aligned}
$$

Furthermore,

$$
t_{\beta_{2}^{\perp}=0}=\frac{y_{-1}^{2^{\prime}} M_{T} \varepsilon\left(\left(y_{-1}^{2^{\prime}} M_{T} y_{-1}^{2}\right)^{3 / 2}\left(y_{-1}^{3^{\prime}} M_{T} y_{-1}^{3}\right)-\left(y_{-1}^{2^{\prime}} M_{T} y_{-1}^{2}\right)^{1 / 2}\left(y_{-1}^{5 / 2^{\prime}} M_{T} y_{-1}^{5 / 2}\right)^{2}\right)}{\hat{\sigma}\left(\left(y_{-1}^{2^{\prime}} M_{T} y_{-1}^{2}\right)^{2}\left(y_{-1}^{3^{\prime}} M_{T} y_{-1}^{3}\right)-\left(y_{-1}^{2^{\prime}} M_{T} y_{-1}^{2}\right)\left(y_{-1}^{5 / 2^{\prime}} M_{T} y_{-1}^{5 / 2}\right)^{2}\right)}
$$




$$
=\frac{y_{-1}^{2^{\prime}} \varepsilon\left(\left(y_{-1}^{2^{\prime}} y_{-1}^{2}\right)^{3 / 2}\left(y_{-1}^{3^{\prime}} y_{-1}^{3}\right)-\left(y_{-1}^{2^{\prime}} y_{-1}^{2}\right)^{1 / 2}\left(y_{-1}^{5 / 2^{\prime}} y_{-1}^{5 / 2}\right)^{2}\right)}{\hat{\sigma}\left(\left(y_{-1}^{2^{\prime}} y_{-1}^{2}\right)^{2}\left(y_{-1}^{3^{\prime}} y_{-1}^{3}\right)-\left(y_{-1}^{2^{\prime}} y_{-1}^{2}\right)\left(y_{-1}^{5 / 2^{\prime}} y_{-1}^{5 / 2}\right)^{2}\right)}+o_{p}(1)
$$

which, as we have shown before, has the asymptotic distribution given in Theorem 1. Finally, among similar lines in the foregoing proof, it is easily seen that the $\tau$ test is consistent under the alternative.

Acknowledgements I would like to thank Jörg Breitung, Philipp Sibbertsen and the participants of the Research Seminar 2007 at the Department of Economics, Hannover and Statistical Week 2007 in Kiel for their helpful comments.

\section{References}

1. Abadir, K.M., Distaso, W., Testing joint hypotheses when one of the alternatives is one-sided, Journal of Econometrics, 140, 695-718 (2007)

2. Dickey, D.A., Fuller, W.A., Distribution of the Estimators for Autoregressive Time Series With a Unit Root, Journal of the American Statistical Association, 74, 427-431 (1979)

3. Dumas, B., Dynamic Equilibrium and the Real Exchange Rate in a Spatially Separated World, Review of Financial Studies, 5, 153-180 (1992)

4. Hamilton J.D., Time Series Analysis. Princeton University Press, Princetion (1994)

5. Hansen, B.E., Convergence to Stochastic Integrals for Dependent Heterogeneous Processes, Econometric Theory, 8, 489-500 (1992)

6. Kapetanios, G., Shin, Y. and A. Snell, Testing for a unit root in the nonlinear STAR framework, Journal of Econometrics, 112, 359-379 (2003)

7. Luukkonen, R., Saikkonen, P. and T. Teräsvirta, Testing linearity against smooth transition autoregressive models, Biometrika, 75, 491-499 (1988)

8. Michael, P., Nobay, A.R. and D.A. Peel, Transactions Costs and Nonlinear Adjustment in Real Exchange Rates: An Empirical Investigation, Journal of Political Economy, 1997, 105, 862-879 (1997)

9. Phillips, P.C.B., Perron, P., Testing for a unit root in time series regression, Biometrika, 75, 335-346 (1988)

10. Phillips, P.C.B., Sul, D., Dynamic panel estimation and homogeneity testing under cross section dependence, Econometrics Journal, 6, 217-259 (2003)

11. Rapach, D. E., Wohar, M. E., The out-of-sample forecasting performance of nonlinear models of real exchange rate behavior, International Journal of Forecasting, 22, 341-361 (2006)

12. Rothe, C., Sibbertsen, P., Phillips-Perron-type unit root tests in the nonlinear ESTAR framework, Allgemeines Statistisches Archiv, 90, 439-456 (2006)

13. Sandberg, R., Convergence to stochastic power integrals for dependent heterogeneous processes, forthcoming in Econometric Theory (2008)

14. Sarantis, N., Modeling non-linearities in real effective exchange rates, Journal of International Money and Finance, 18, 27-45 (1999)

15. Sercu, P., Uppal, R. and C. Van Hulle, The Exchange Rate in the Presence of Transaction Costs: Implications for Tests of Purchasing Power Parity, Journal of Finance, 50 1309-1319 (1995)

16. Taylor, M.P., Peel, D.A. and L. Sarno, Nonlinear Mean-Reversion in Real Exchange Rates: Toward a Solution To the Purchasing Power Parity Puzzles, International Economic Review, 42, 1015-1042 (2001)

17. Teräsvirta, T., Specification, estimation and evaluation of smooth transition autoregressive models, Journal of the American Statistical Association, 89, 208-218 (1994) 